\title{
A method for determination of mass-exchange and capacitive characteristics of absorbers for life support systems
}

\author{
Pavel V. Balabanov ${ }^{1, *}$, Guzel R. Kamaletdinova ${ }^{1}$, and Maria G. Muravyeva ${ }^{1}$ \\ ${ }^{1}$ Tambov State Technical University, 392000 Tambov, Sovetskaya 106, Russia
}

\begin{abstract}
We describe a method for determining mass-exchange and capacitance characteristics of chemisorbents of carbon dioxide. Through a cartridge equipped with a chemosorbent, a gas-air mixture is blown continuously from a sealed container of a small volume into which the impurity of carbon dioxide is continuously introduced. The gas-air mixture, which has been purified from the impurity, is again introduced into the tank. The concentration of carbon dioxide in a sealed volume is monitored by means of the gas analyzer; the effective mass transfer coefficient and the maximum capacity of the chemisorbent for carbon dioxide are calculated from the obtained data.
\end{abstract}

The development of methodology for certification of parameters of regeneration processes for the maximum capacity of chemisorbents, mass exchange coefficients for carbon dioxide and oxygen for substances used in life support systems is an urgent task [1-5]. Previously, theoretical aspects of the dynamics of chemisorption by regenerative substances were developed in [1], mathematical models for the functioning of collective and individual protective equipment, on the basis of which the parameters of air regeneration processes were determined in $[2,3]$. Methods and equipment for the automated investigation of the characteristics of chemisorbents were proposed in $[4,5]$.

It is noteworthy that parameter identification was carried out on the basis of rather complex mathematical models, containing a system of equations in partial derivatives using numerical calculation methods, which required the creation of special software [6-10].

At the same time, the practice of certification of devices for air regeneration has shown that there is a need for an automated method for determining the mass exchange and capacitance characteristics of chemisorbents (absorbers) that would be universal for substances and cartridges of any configuration.

The purpose of this paper is to develop such a method.

We consider a life-support system with low volume $V$ to $100 \mathrm{dm}^{3}$, for which the exchange multiplicity $Q / V$ ( $Q$ is the volume of ventilation) is so large that the influence of the volume $V$ on the concentration of carbon dioxide can be neglected.

The equation for the dynamics of chemisorption $\mathrm{CO}_{2}$ can be written in the form

$$
\frac{\partial \varphi}{\partial \tau}+\frac{\partial C}{\partial \tau}=-w \frac{\partial C}{\partial x}+D \frac{\partial^{2} C}{\partial x^{2}},
$$

where $\varphi, C$ are current absorption and concentration of dioxide in the gas phase, respectively, $x$ is the current coordinate counted from the inlet of the gas flow to the chemisorbent layer, $D$ is coefficient of longitudinal diffusion, $w$ is linear velocity, $\tau$ is time.

We assume that the cartridge is filled with chemosorbent, the process is irreversible and its velocity is determined by a universal equation of the form $[1,2$, $5,11]$

$$
\partial \varphi / \partial \tau=\beta C\left(1-\varphi / \varphi_{0}\right)
$$

where $\varphi_{0}$ is end capacity for carbon dioxide; $\beta$ is mass transfer coefficient.

An approximate allowance for the irregular transfer of the absorbed component in the flow within the layer can be carried out by the method proposed in [12]. In this case, the effect of a longitudinal transfer may be taken into account by an effective mass transfer coefficient

$$
\beta_{\mathrm{ef}} \approx k \beta \text {, }
$$

where

$$
k \in\left[k_{1} ; k_{2}\right], k_{1}=\frac{1}{1+y}, k_{2}=\frac{2}{1+\sqrt{1+4 y}}, y=D \beta / w^{2} .
$$

The initial conditions are assumed to be zero

$$
C(x, 0)=\varphi(x, 0)=0 .
$$

To determine the boundary conditions, we assume that the concentration $C_{0}$ existing at the initial time is defined as the quotient obtained by load division for impurities $q$ by the total volume of ventilation

\footnotetext{
* Corresponding author: pav-balabanov@yandex.ru
} 


$$
C_{0}=q / Q
$$

The concentration of carbon dioxide at the inlet to the cartridge is an unknown function of time and is composed of a constant concentration $C_{0}$ due to the presence of a source of impurity release and concentration at the outlet of the cartridge $C_{0} \Psi(\tau)$. We assume that the flow of carbon dioxide in the volume starts with the time $\tau=L / w$, where $L$ is sorbent bed length. At the inlet to the cartridge there exists concentration $C_{0}+C_{0} \Psi(\tau)$. The amount of carbon dioxide released in time $d \tau$ from the cartridge is equal to $S w C_{0} \Psi(\tau) d \tau$, and the amount of impurity (carbon dioxide) supplied to the cartridge (carbon dioxide) is $S w C_{0}(1+\Psi(\tau)) d \tau$.

Usually the device is used to the maximum permissible concentrations, for example, of carbon dioxide. If the initial concentration $\mathrm{CO}_{2}$ is $0.5 \%$ (in this case $q=30 \mathrm{dm}^{3} / \mathrm{h}, Q=6 \mathrm{~m}^{3} / \mathrm{h}$ ), the end concentration $\mathrm{CO}_{2}$ is $3.0 \%$, then for the volume of $100 \mathrm{dm}^{3}$ accumulation of $\mathrm{CO}_{2}$ must not exceed $2.5 \mathrm{dm}^{3}$. The latter means that the device (cartridge) must absorb practically the entire volume of carbon dioxide fed into the life support system during the time of its actual operation.

Thus, the amount absorbed in the cartridge in a time $\mathrm{d} \tau$

$$
d \tau S \int_{0}^{L} \frac{\partial(\varphi+C)}{\partial \tau} d x=-d \tau S \int_{0}^{L} w \frac{\partial C}{\partial x} d x \approx S w C_{0} d \tau
$$

Hence

$$
C_{0} \approx-\int_{0}^{L} \frac{\partial C}{\partial x} d x .
$$

Given the dimensionless variables

$$
u=\frac{C}{C_{0}}, \xi=\frac{\beta_{\mathrm{ef}} x}{w}, t=\frac{\beta_{\mathrm{ef}} C_{0}}{\varphi_{0}}\left(\tau-\frac{x}{w}\right),
$$

from equation (6) we obtain

$$
1 \approx-\int_{0}^{\xi_{L}} \frac{\partial u}{\partial \xi} d \xi
$$

We note that under the usual operating conditions of the cartridge the condition

$$
-w \int_{0}^{\xi_{L}} \frac{\partial C}{\partial \xi} d \xi>>\int_{0}^{\xi_{L}} \frac{\partial C}{\partial t} d \xi
$$

is satisfied.

We use the expression for the distribution of the dimensionless concentration of the absorbed substances in the mobile phase inside the device obtained by solving the system of equations (1), (2) with the initial condition $\varphi(x, 0)=$ const and boundary condition for concentration
$C(0, \tau)=C_{0} \Psi(\tau)$. The solution of this problem has the form

$$
u=\frac{\Psi(t)}{e^{-\int^{t} \Psi(t) d t}\left[e^{\xi}-1\right]+1} .
$$

Differentiating the last expression with respect to $\xi$ and substituting the result in (7), we obtain

$$
1=\int_{0}^{\xi} \frac{\Psi(t) \exp \left(\int_{0}^{t} \Psi(t) d t\right) \exp \xi}{\left[\exp \left(\int_{0}^{t} \Psi(t) d t\right)+e^{\xi}-1\right]^{2}} d \xi .
$$

After integrating expression (10) with respect to $\xi$ and making simple transformations, we obtain

$$
\exp \left(\int_{0}^{t} \Psi(t) d t\right)=[\Psi(t)-1]\left[\exp \xi_{L}-1\right] .
$$

The logarithm and differentiation of the resulting expression gives an equation for finding $\Psi(\tau)$

$$
\Psi^{2}(t)=\Psi^{\prime}(t)+\Psi(t),
$$

which by substituting $\Psi(\tau)=1 / z$ is reduced to the linear equation having a solution

$$
\Psi(t)=\frac{\exp (-t)}{\exp (-t)+\text { Const }} .
$$

where const $=\exp \left(-\xi_{L}\right)$ is found by substituting the last expression into equation (11). Consequently,

$$
\Psi(t)=\frac{\exp \left(t-\xi_{L}\right)}{1-\exp \left(t-\xi_{L}\right)} .
$$

Expression (14) is applicable to $t<\xi_{L}$. Passing to dimensional variables, after simple transformations we obtain equation

$$
\tau=\frac{\varphi_{0}}{w C_{0}} L+\frac{\varphi_{0}}{\beta_{\mathrm{ef}} C_{0}} \ln \frac{C}{C_{0}+C},
$$

that is the basis of the method for determining the massexchange and capacitive characteristics of sorbents.

For a cartridge with a cylindrical layer and a radial flow direction: $W=Q /(2 \pi h), L=\left(R_{1}^{2}-R_{2}^{2}\right) / 2$, where $h$ is the height of a cylindrical layer, $R_{1}, R_{2}$ are large and small radii of the cylindrical layer, respectively.

Proposed method can be represented as a sequence of the following operations.

The absorbent cartridge 3 is filled with the chemosorbent under the study, then the cartridge inlet is connected to the outlet of the flow agitator (Fig. 1). The outlet of the cartridge is connected with the flange fitting of the tank1 having the volume $V$. Then the fan and the agitator for blowing gas-air mixture from the tank 1 through the cartridge with a constant flow $Q$ and 
temporary flow $q$ are turned on. From cylinder 2 carbon dioxide is fed into the tank, simulating the load on the device. The gas-air mixture purified from carbon dioxide is returned to the volume $\mathrm{V}$, where it is mixed with $\mathrm{CO}_{2}$ newly supplied by the fan. During the test, the concentration $C$ of carbon dioxide in the tank 1 , the consumption of gas-air mixture flown through the cartridge and the consumption of $\mathrm{CO}_{2}$ from the cylinder are recorded. Based on the experimental data obtained, the parameters $\alpha_{1}$ and $\alpha_{2}$ are found

$$
\tau=\alpha_{1} \ln \frac{C}{C_{0}+C}+\alpha_{2}
$$

where $\tau$ is current time, $C_{0}, C$ are initial and current concentrations of $\mathrm{CO}_{2}$ in the tank 1.

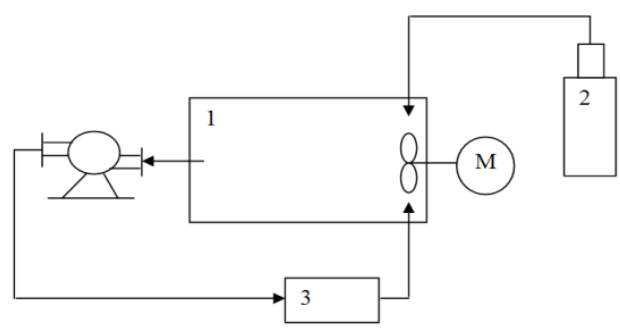

Fig. 1. Installation diagram for testing

The effective mass transfer coefficient and the limiting capacity for carbon dioxide under the experimental conditions are found from the expressions

$$
\beta_{\mathrm{ef}}=\frac{\varphi_{0}}{\alpha_{1} C_{0}}, \varphi_{0}=\frac{\alpha_{2} w C_{0}}{L} .
$$

As an example, we give the results of tests of absorbers based on $\mathrm{LiOH}$ in the model of a cartridge of cylindrical shape with a diameter of $117 \mathrm{~mm}$. In the experiments, we used chemisorbents in the form of cylindrical blocks with channels. The length of the layer, depending on the thickness of the test blocks, varied in the range $195 \ldots 210 \mathrm{~mm}$. On average, the multiplicity of transfer $Q / V$ was equal to $18 \mathrm{~h}^{-1}$. The supply of carbon dioxide was varied in such a way that $q / Q$ was within the range of $0.0107 \ldots 0.0124$. The tests were carried out at room temperature of the medium and the relative humidity of gas-air mixture of $80 \pm 10 \%$. Fig. 2 shows the curves of changes in the concentration of carbon dioxide in time; the results were obtained in the two experiments with the absorber blocks. In the first experiment (curve 1) the mass of each block was 1.12 times larger than that in the second one (curve 2). Fig. 3 shows the experimental curves plotted in semilogarithmic coordinates.

The results of finding mass transfer characteristics of the investigated absorbers are given in the Table 1.

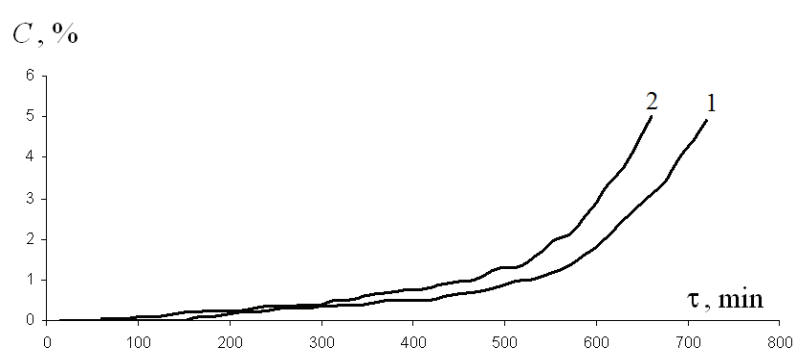

Fig. 2. Time variation in concentration

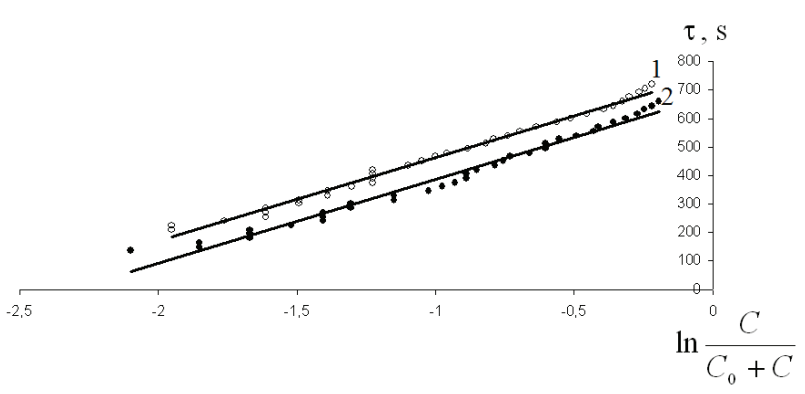

Fig. 3. Experimental curves in semilogarithmic coordinates

Table 1. The results of the determination of the mass transfer characteristics of the investigated absorbers

\begin{tabular}{|c|c|c|c|c|c|}
\hline \multirow{2}{*}{$\begin{array}{c}\text { № } \\
\text { test }\end{array}$} & \multicolumn{2}{|c|}{ Process characteristics } & \multicolumn{2}{c|}{$\begin{array}{c}\text { Mass transfer } \\
\text { characteristics }\end{array}$} \\
\cline { 2 - 6 } & $\begin{array}{c}Q / V, \\
\mathrm{~h}^{-1}\end{array}$ & $q / Q$ & $L, \mathrm{~m}$ & $\begin{array}{c}\beta_{e f}, \\
1 / \mathrm{s}\end{array}$ & $\begin{array}{c}\varphi_{0}, \\
\mathrm{~m}^{3} / \mathrm{m}^{3}\end{array}$ \\
\hline 1 & 18 & 0,0124 & 210 & 1,23 & 260 \\
\hline 2 & 18 & 0,0107 & 195 & 1,18 & 224 \\
\hline
\end{tabular}

The root-mean-square errors in the determination of mass transfer characteristics calculated by the formulas

$$
\begin{gathered}
\left(\frac{\Delta \varphi_{0}}{\varphi_{0}}\right)_{\mathrm{sc}}=\sqrt{\left(\frac{\Delta \alpha_{2}}{\alpha_{2}}\right)^{2}+\left(\frac{\Delta w}{w}\right)^{2}+\left(\frac{\Delta C_{0}}{C_{0}}\right)^{2}+\left(\frac{\Delta L}{L}\right)^{2}} \\
\left(\frac{\Delta \beta_{\mathrm{ef}}}{\beta_{\mathrm{ef}}}\right)_{\mathrm{sc}}=\sqrt{\left(\frac{\Delta \alpha_{1}}{\alpha_{1}}\right)^{2}+\left(\frac{\Delta \varphi_{0}}{\varphi_{0}}\right)^{2}+\left(\frac{\Delta C_{0}}{C_{0}}\right)^{2}},
\end{gathered}
$$

did not exceed $7 \%$ for $\varphi_{0}$ and $9 \%$ for $\beta_{e f}$. In the calculations, we took into account that the errors in measuring the consumption of carbon dioxide $q$ did not exceed $1 \%$, of the gas-air mixture consumption $Q$ did not exceed $2.5 \%$, the layer length and the block diameters did not exceed 2\%. Parameters $\alpha_{1}$ and $\alpha_{2}$ were determined by approximating the dependences shown in Fig. 3 as the equation of a straight line. In order to increase the accuracy, we used only the experimental data for which condition $C>0,2$ was satisfied because at lower concentrations the consumption of carbon dioxide and gas-air mixture can vary, and the errors in the measurement of concentrations can occur. Errors in finding $\alpha_{1}$ and $\alpha_{2}$ did not exceed 5\%. The root-mean- 
square errors of $C_{0}$, calculated by (4) did not exceed $3 \%$, the velocity of gas-air mixture flow calculated by the known consumption $Q$, did not exceed $3 \%$.

We note that the obtained values $\beta$ and $\varphi_{0}$ for lithium absorber blocks were different from those given in [2]. Thus, in particular, the limiting capacity for $\mathrm{CO}_{2}$, equal to $216 \ldots 260 \mathrm{~m}^{3} / \mathrm{m}^{3}$ was close to the stoichiometric under normal conditions (temperature 0 and pressure $760 \mathrm{~mm} \mathrm{Hg}$ ). This is due to the difference in test conditions (mainly for concentrations $\mathrm{CO}_{2}$ at the inlet to the layer). With a decrease in the supply of carbon dioxide to the volume, the values $\beta$ and $\varphi_{0}$ will approach the data given in [2], but the total duration of the experiment will increase substantially.

Thus, we proposed an "express" method, which allows determining the chemisorption characteristics of the absorbers relatively quickly and in a wide range of impurity concentrations. The method can be used in the study of the quality of carbon dioxide absorbers, as well as in the certification of absorption devices for life support systems.

Financial state support from Russian Scientific Foundation (Project15-19-10028 "The development of the theoretical foundations for creating the research base for human respiratory protection systems from the chemical and biological dangerous nature factors") on the base of Tambov State Technical University is gratefully acknowledged.

\section{References}

1. S.V. Mishchenko, P.V. Balabanov, A.A. Krimshtejn, S.V. Ponomarev, Trans. TSTU, 16, 870 (2010)

2. A.A. Krimshtein, S.V. Plotnikova, V.I. Konovalov, B.V. Putin, J. Appl. Chem. USSR, 65, 2031 (1992)

3. A.A. Krimshtein, S.V. Plotnikova, V.I. Konovalov, B.V. Putin, Rus. J. Appl. Chem., 66, 1356 (1993)

4. P.V. Balabanov, A.A. Krimshtejn, S.V. Ponomarev, Ind. Labor. Diagn. Mater., 77, 34 (2011)

5. S.B. Putin, S.V. Gudkov, E.N. Tugolukov, N.P. Kozlova, S.Yu. Alekseev, A.Yu. Khromov, Trans. TSTU, 14, 90 (2008)

6. J. Li, L. Jin, S. Wang, Z. Zhang, Y. Xu, Q. Li, Adv. Mater. Res., 726, 363 (2013)

DOI:10.4028/www.scientific.net/AMR.726-731.363

7. J.H. Kim, Y.K. Park, S.K. Jeong, Korean J. Chem. Eng., 27, 320 (2010)

DOI: $10.1007 / \mathrm{s} 11814-009-0291-7$

8. S. Choi, J.H. Drese, C.W. Jones, ChemSusChem, 2, 796 (2009) DOI: 10.1002/cssc.200900036

9. S. Wang, S. Yan, X. Ma, J. Gong, Energy Environ. Sci., 4, 3805 (2011) DOI: 10.1039/c1 ee01116b

10. C.-H. Yu, C.-H. Huang, C.-S. Tan, Aerosol Air Qual. Res., 12, 745 (2012)

DOI: 10.4209/aaqr.2012.05.0132

11. A.M. Kudryavtsev, A.V. Kudryavtseva, A.A. Krimshtejn, S.V. Plotnikova, Trans. TSTU, 1, 59 (1995)
12. V.L. Kolin, G.I. Bagrov, G.A. Musakin, V.P. Semenov, in Kinetics and dynamics of physical adsorption, 98 (Nauka, Moscow, 1973) 